\title{
Corrigendum: Design of a genome-wide siRNA library using an artificial neural network
}

Dieter Huesken, Joerg Lange, Craig Mickanin, Jan Weiler, Fred Asselbergs, Justin Warner, Brian Meloon, Sharron Engel, Avi Rosenberg, Dalia Cohen, Mark Labow, Mischa Reinhardt, François Natt \& Jonathan Hall

Nat. Biotechnol. 23, 995-1001 (2005)

On page 1,000, in the last Methods section, line 4 from bottom of page, "HeLa cells were treated with 100 mM Deferoxamine Mesylate" should (

In the supplementary online information, the original Supplementary Table 3 erroneously showed the Biopred algorithm scores (predicted relative activity) in the last column, instead of experimentally determined 'Normalized inhibitory activity'. This has been corrected in the new Supplementary Table 3 and the appropriate footnotes added. The changes affect all of the $\sim 3,000$ values listed in the last column of the table.

\section{Corrigendum: Overexpression of an Arabidopsis thaliana ABC transporter confers kanamycin resistance to transgenic plants}

Ayalew Mentewab \& C Neal Stewart Jr

Nat. Biotechnol. 23, 1177-1180 (2005)

On p. 1180, line 4, the reverse primer to amplify the gene encoding Atwbc19 was given as ( $5^{\prime}$-TGTCCCCGTTTTTATCCAAG-3'). The reverse primer used was 5'-GTCAGCGAGCTCTCACGTCCTCTTATTCCTGC-3', which contains a SacI site.

\section{Erratum: Chasing a cellular fountain of youth}

Ken Howard Wilan, Christopher Thomas Scott \& Stephan Herrera

Nat. Biotechnol. 23, 807-815 (2005)

On page 808, in Table 1, the entry for MacroPore Biosurgery (which since the publication of this article has changed its name to Cytori Therapeutics) erroneously listed "liver-assist device (with subsidiary Xenogenics)" after "Cell-based therapies, bioresorbable therapies" in the business model column. It should have read simply, "Cell-based therapies, bioresorbable therapies." 